\title{
Comparison of nutrient intakes by nutritional anemia and the association between nutritional anemia and chronic diseases in Korean elderly: Based on the 2013-2015 Korea National Health and Nutrition Examination Survey Data
}

\author{
So Hyun Park, So Hee Han and Kyung Ja Chang ${ }^{\text {\$ }}$ \\ Department of Food and Nutrition, Inha University, 100 Inha-ro, Michuhol-Gu, Incheon 22212, Korea
}

BACKGROUND/OBJECTIVES: The elderly are reported to have a high prevalence of nutritional anemia when they have lower intakes of nutrients or chronic diseases. This study was conducted to compare nutritional status according to nutritional anemia and to determine associations between nutritional anemia and chronic diseases in Korean elderly.

SUBJECTS/METHODS: This study utilized data on 3,258 elderly aged $\geq 65$ years gathered during the $6^{\text {th }}$ Korea National Health and Nutrition Examination Survey 2013-2015. Subjects were divided into nutritional anemia (NA) group ( $n=415)$ and non-NA group $(n=2,843)$ by hemoglobin concentration. Nutrient intakes were assessed using dietary intake data obtained using the 24-hour recall method. The odds ratios (ORs) for nutritional anemia by chronic diseases were determined. Statistical analysis was performed using SPSS Ver. 23.0.

RESULTS: Of 3,258 subjects, $12.7 \%$ had nutritional anemia. Intakes of potatoes, pulses, and mushrooms by males and potatoes, fruits, meats, eggs, and seafood by females were significantly lower in NA group than in non-NA group. The proportion of the subjects whose intakes of protein, vitamin $A$, vitamin $B_{1}$, vitamin $B_{2}$, niacin, and iron less than estimated average requirement (EAR) were significantly higher in NA group compared to non-NA group. After adjusting for age, the number of family members, energy intake, and alcohol drinking, ORs for nutritional anemia in the subjects with diabetes and myocardial infarction or angina pectoris were significantly higher by 1.74 times and 1.59 times as compared to the subjects without those diseases, respectively. However, ORs for nutritional anemia in the subjects with obesity, abdominal obesity, and hypertriglyceridemia were significantly lower by 0.64 times, 0.60 times, and 0.59 times as compared to the subjects without those diseases, respectively. CONCLUSIONS: The results of this study suggested that nutritional management should be done to enable the Korean elderly to consume foods with high hematopoietic nutrients density to prevent nutritional anemia. Korean elderly need to make regular efforts to check for nutritional anemia.

Nutrition Research and Practice 2019;13(6):543-554; https://doi.org/10.4162/nrp.2019.13.6.543; pISSN 1976-1457 elSSN 2005-6168

Keywords: Anemia, Korean elderly, nutrients, chronic disease, hemoglobin

\section{INTRODUCTION}

Nutritional anemia is defined as a condition in which hemoglobin concentration falls below a criteria level due to insufficient storage of hematopoietic nutrients required for the synthesis or metabolism of hemoglobin and erythrocytes [1]. In the United Nations, people aged 65 years and older are classified as the elderly [2], and nutritional anemia in the elderly is determined by a hemoglobin concentration below $13 \mathrm{~g} / \mathrm{dL}$ for male and below $12 \mathrm{~g} / \mathrm{dL}$ for female according to the World Health Organization (WHO) [1,3]. However, generally, hemoglobin concentrations in blood decreased with age, but lower hemoglobin concentrations were reported to be associated with increased risk of hospitalization, morbidity, and mortality $[4,5]$. The prevalence of anemia in the elderly has been reported to be between $8 \%$ and $25 \%$ and to be $20 \%$ more than in those aged 85 years over [6,7].

In the National Health and Nutrition Examination Survey (NHANES, 1991-1994) [6], 34.3\% of the elderly with anemia was caused by nutritional deficiency anemia and in $32.1 \%$ of their anemia was associated with chronic diseases. Hemoglobin concentrations of elderly patients with nutritional anemia were 8 to $10 \mathrm{~g} / \mathrm{dL}$, and usually had mild to moderate anemia. In fact, nutritional anemia in elderly patients accounts for one-third of all anemia [8]. The most common type of nutritional anemia is iron-deficiency anemia, which accounts for $\geq 60 \%$ of cases,

\footnotetext{
This work was supported by a grant from Inha University.

${ }^{\S}$ Corresponding Author: Kyung Ja Chang, Tel. 82-32-860-8126, Fax. 82-32-862-8120, Email. kjchang@inha.ac.kr

Received: May 9, 2019, Revised: June 6, 2019, Accepted: August 29, 2019

This is an Open Access article distributed under the terms of the Creative Commons Attribution Non-Commercial License (http://creativecommons.org/licenses/by-nc/3.0/) which permits unrestricted non-commercial use, distribution, and reproduction in any medium, provided the original work is properly cited.
} 
and its causes are known to be chronic gastrointestinal blood loss caused by drug taking, gastritis, esophagitis, other chronic diseases, impaired efficiency of iron absorption, decreased physical functional capacity, and inflammation. In addition to iron deficiency, nutritional anemia in the elderly is associated with poor intake of folic acid, vitamin $B_{12}$, protein, vitamin $B_{2}$, vitamin $C[6,9]$. In addition, nutritional anemia in the elderly was associated with aging-associated masticatory, deglutition, and digestion disorders [10] and associated with meal skipping or the lack of micronutrient intakes due to living alone with age [11]. Percentage of elderly is rapidly increasing worldwide (8.2\% in 2015 and predicted to increase to $11.6 \%$ in 2030) [12], so the prevalence of nutritional anemia is expected to increase continuously in the elderly and to pose a considerable healthcare burden. Recently in Korea, for example, the prevalence of nutritional anemia in 2011 and 2016 were $7.4 \%$ and $8.0 \%$, respectively, $16.8 \%$ for those in their $60 \mathrm{~s}$ and $17.9 \%$ for those in their $70 \mathrm{~s}$ [13].

The elderly population ratio in Korea was $14.3 \%$ in 2018 and is expected to exceed $20 \%$ in 2025 and the speed rate of population aging is greatest in OECD countries [12,14]. In Korea, life expectancy increased from 64.6 years in 1976 to 82.4 years in 2016, but healthy span, which now stands at 64.9 years, has shown little change over the past 40 years [15]. In 2017, about $90 \%$ of Korean elderly had a chronic disease, $73 \%$ of them had two or more chronic diseases [16], and in about 33\% of Korean elderly with chronic disease were accompanied by nutritional anemia [17]. In elderly patients with chronic disease, nutritional anemia can lead to health problems such as aggravated symptoms of chronic disease, increased mortality, and decreased exercise resulting in decreased muscle mass [18]. Thus, the study of nutritional anemia in Korean elderly should be considered inadequate nutrient intakes and chronic diseases.

Although nutritional anemia has become an important health problem in Korea due to population aging, few studies have investigated its relations with nutrient intakes status and chronic diseases according to nutritional anemia in the elderly. Therefore, the purpose of this study was conducted to investigate the associations between nutritional anemia and nutrient intakes status and chronic diseases in Korean elderly by analyzing data collected the $6^{\text {th }}$ Korea National Health and Nutrition Examination Survey (KNHANES) 2013-2015.

\section{SUBJECTS AND METHODS}

\section{Study population and design}

This study used data from the $6^{\text {th }}$ KNHANES (2013-2015) conducted by the Korea Centers for Disease Control and Prevention (KCDC) of the Ministry of Health and Welfare. This survey was approved by the institutional review board (IRB) of the KCDC (IRB approval number in 2013 and 2014: 201307CON-03-4C and 2013-12EXP-03-5C). Following the Bioethics and Safety Act in Korea, KNHANES as the study conducted by the government for public welfare has been conducted without any review by IRB since 2015. Raw data used in this study were obtained on May 30, 2018, after obtaining approval from the KNHANES website.

As shown in Fig. 1, 3,258 participants aged 65 years and older were selected from the 22,948 participants that participated in $6^{\text {th }}$ KNHANES; 18,439 participants were excluded for age under 65, 183 participants for missing data on nutrient intakes, 46 participants for reporting implausible dietary intakes $(<500 \mathrm{kcal}$

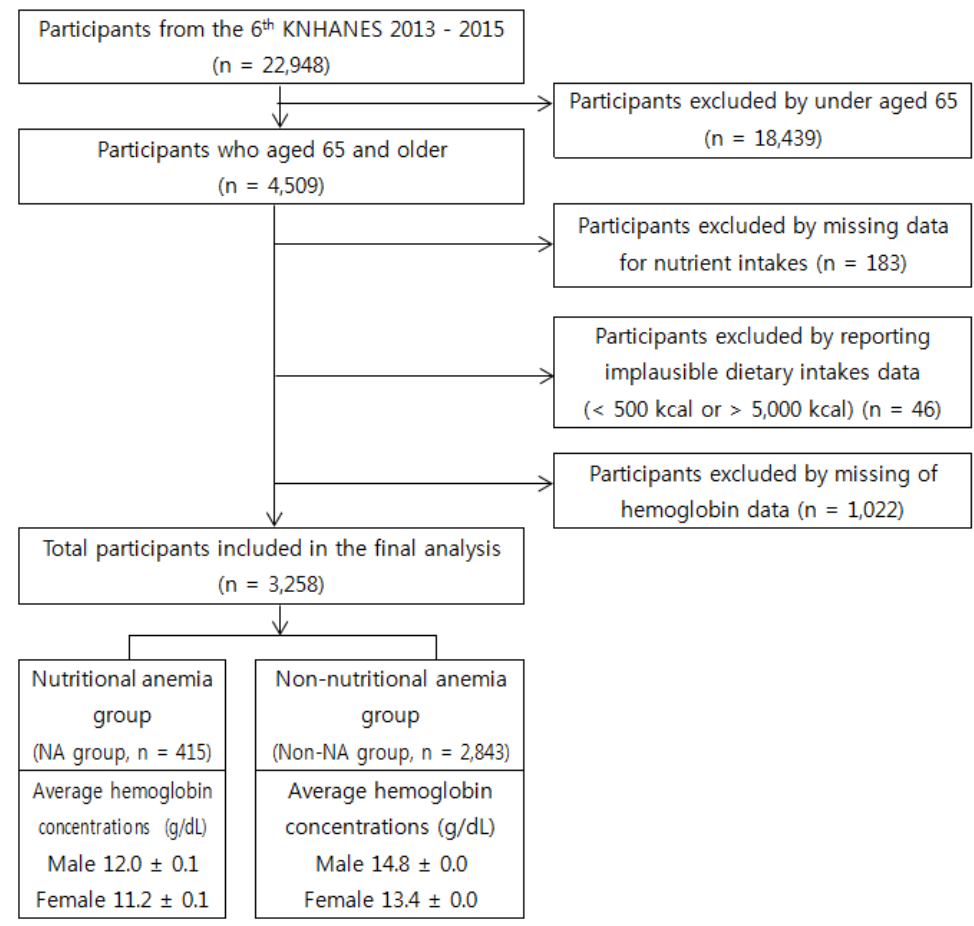

Fig. 1. Flow chart of participants included in the analysis 
or $>5,000 \mathrm{kcal}$ per day) [19], and 1,022 participants for no hemoglobin data. Nutritional anemia group (NA group) and non-NA group were classified by hemoglobin concentration in blood ( $<13 \mathrm{~g} / \mathrm{dL}$ for male and $<12 \mathrm{~g} / \mathrm{dL}$ for female) [2]. Hemoglobin concentration in blood was determined by using an XE-2100D analyzer (Sysmex, Japan) in 2013-2014 and an XN-9000 analyzer (Sysmex, Japan) in 2015. Average hemoglobin concentrations in blood of male and female subjects were 12.0 $\mathrm{g} / \mathrm{dL}$ and $11.2 \mathrm{~g} / \mathrm{dL}$ in NA group, $14.8 \mathrm{~g} / \mathrm{dL}$ and $13.4 \mathrm{~g} / \mathrm{dL}$ in non-NA group, respectively $(P<0.001)$. Thus, subjects were divided into 415 subjects with nutritional anemia (12.7\%) in NA group and 2,843 subjects without nutritional anemia (83.7\%) in non-NA group.

\section{General characteristics}

General characteristics were obtained from gender, age, region, educational level, income level, and the number of family members included in the basic variables data of KNHANES. Age groups were classified as 65 to 74 years and aged 75 years and older $[2,20]$. Region was classified as urban or rural, and educational level was classified as elementary school or less, middle school, high school, or college graduate or more. Income level was divided into quartiles by mean values of age and gender subgroups, and the number of family members was classified as single or more than two persons.

\section{Anthropometric and blood pressure data}

Anthropometric data were obtained from values of height, body weight, waist circumference, and body mass index (BMI) included in the anthropometric measurement data of KNHANES. Height, body weight, and waist circumference were measured using extensometer (SECA 200, SECA, Germany), body weight scale (GL-6000-20, G-TECH, Korea), and tape measure (SECA 200, SECA, Germany), respectively. BMI was calculated by dividing body weight $(\mathrm{kg})$ by height squared $\left(\mathrm{m}^{2}\right)$.

Blood pressure data were obtained from values of final systolic and diastolic blood pressure (average values of the second and third blood pressure measurements taken over 3 separate sessions) included in blood pressure measurement data of KNHANES. Blood pressure was measured using a sphygmomanometer (Baumanometer Wall Unit 33, Baum, USA) after a five-minute rest.

\section{Biochemical data in blood}

Biochemical data in blood were obtained from levels of fasting blood glucose and serum lipid profiles (total cholesterol, TC; high-density lipoprotein cholesterol, HDL-C; triglyceride, TG; low-density lipoprotein cholesterol, LDL-C) included in blood test of KNHANES. Blood was obtained from participants in the morning after at least 8-hour of fasting overnight. Fasting blood glucose, serum lipid, and serum creatinine levels were determined by using a Hitachi Automatic Analyzer 7600-210 (Hitachi, JAPAN). Blood analyses were performed by the Neodin Medical Institute; a laboratory certified by the Korea Centers for Disease Control \& Prevention.

\section{Dietary intake assessment}

Intakes of major food groups, energy, nutrients, and dietary fiber were obtained from values included in the nutrition survey of the KNHANES. Survey of dietary intake was completed by using a 24-hour recall method by a face-to-face interview with trained staff. Major food groups were categorized into 17 items based on the Korean Food Composition Table $9^{\text {th }}$ Revision by the Rural Development Administration [21]. Intakes of energy, nutrients, and dietary fiber were assessed and the proportions of the subjects consuming nutrients less than the EAR using the 2015 dietary reference intakes for Korean (KDRIs) were compared according to nutritional anemia [20].

\section{Health-related lifestyle habits}

Health-related lifestyle habits were obtained from values included in the nutrition survey of the KNHANES. They included alcohol drinking, smoking status, and walking frequency. Alcohol drinking, smoking status, and walking frequency were assessed using yes or no responses to 'less than 1 glass/month', 'current smoking', and 'more than 30 min per day and 5 days per week', respectively.

\section{Criteria for the diagnosis of chronic diseases}

Chronic diseases in this study included obesity as determined by $\mathrm{BMI}$, abdominal obesity, hypertension, diabetes, hypercholesterolemia, hypertriglyceridemia, myocardial infarction or angina, and osteoarthritis or rheumatoid arthritis [22]. Based on criteria in the guidebook for the KNHANES Raw Data, these chronic diseases were defined [23]. Obesity as determined by BMI and abdominal obesity were defined as BMl of $\geq 25.0 \mathrm{~kg} / \mathrm{m}^{2}$ and waist circumference of $\geq 90 \mathrm{~cm}$ for male and $\geq 85 \mathrm{~cm}$ for female. Hypertension was defined as systolic or diastolic blood pressure of $\geq 140$ or $90 \mathrm{mmHg}$, respectively, or taking of hypertension medication. Diabetes was defined as fasting blood glucose level of $\geq 126 \mathrm{mg} / \mathrm{dL}$ or taking of diabetes medication. Cholesterolemia, low HDL cholesterolemia, hypertriglyceridemia, and high LDL cholesterolemia were defined as TC level of $\geq$ $240 \mathrm{mg} / \mathrm{dL}$ or taking of cholesterol-lowering drugs, as HDL-C level of $\leq 40 \mathrm{mg} / \mathrm{dL}$, as TG level of $\geq 200 \mathrm{mg} / \mathrm{dL}$, and as LDL-C level of $\geq 130 \mathrm{mg} / \mathrm{dL}$, respectively. Myocardial infarction or angina pectoris, osteoarthritis or rheumatoid arthritis, and kidney failure were diagnosed with the presence of them depending on diagnosis by a doctor.

\section{Statistical analysis}

Because the KNHANES data were collected using a complex sampling design involving cluster and stratified samplings, $6^{\text {th }}$ KNHANES data were analyzed by applying primary sampling units (PSU), strata (Kstrata), and integrated weights. Continuous variables presented as mean \pm standard error (SE) and categorical variables presented as percentage and SE were verified for significance using complex sample general linear model t-test and complex sample chi-square test according to the prevalence of nutritional anemia, respectively. Relationships between nutritional anemia and nutrient intakes and chronic diseases were analyzed using multiple logistic regression analysis. This analysis was performed to obtain the odds ratio (OR) and 95\% confidence interval $(\mathrm{Cl})$ adjusted for confounding variables. Three models were presented in ways as follows: Model 1 was not adjusted for confounding variables; Model 2 was adjusted 
for age and the number of family members; Model 3 was adjusted for age, the number of family members, energy intake, and alcohol drinking.

Statistical analyses of data were conducted using IBM SPSS Statistics for Windows version 23.0 program (Armonk, NY, USA: IBM Corp.), and the level of statistical significance levels was set at $P<0.05$.

\section{RESULTS}

General characteristics of the subjects by nutritional anemia

General characteristics of the subjects by the presence of nutritional anemia are shown in Table 1. Percentage of male and female subjects was $43.4 \%$ and $56.6 \%$ in NA group and $45.2 \%$ and $54.8 \%$ in non-NA group, respectively. Percentages of the subjects aged 65 to 74 years and $\geq 75$ years were $56.8 \%$ and $43.2 \%$ in NA group (average age 73.3 ) and $70.1 \%$ and $29.9 \%$ in non-NA group (average age 71.7$)$, respectively $(P<0.001)$. For the number of family members, percentage of the subjects living alone in NA and non-NA groups was $26.0 \%$ and $19.2 \%$, respectively $(P<0.01)$.

Anthropometric and blood pressure data of the subjects by nutritional anemia and gender

Differences in anthropometric and blood pressure data in NA and non-NA groups by gender are shown in Table 2. Average $\mathrm{BMI}$ and waist circumference in NA and non-NA groups were $22.6 \mathrm{~kg} / \mathrm{m}^{2}$ and $82.8 \mathrm{~cm}$ and $23.8 \mathrm{~kg} / \mathrm{m}^{2}$ and $86.2 \mathrm{~cm}$ for male subjects $(P<0.001)$ and $23.8 \mathrm{~kg} / \mathrm{m}^{2}$ and $81.5 \mathrm{~cm}$ and $24.5 \mathrm{~kg} / \mathrm{m}^{2}$ and $84.1 \mathrm{~cm}$ for female subjects $(P<0.01)$, respectively. Average diastolic blood pressure in NA group $(66.2 \mathrm{mmHg}$ for males and $68.8 \mathrm{mmHg}$ for females) was significantly lower compared to non-NA group (73.1 $\mathrm{mmHg}$ for males and $72.7 \mathrm{mmHg}$ for females, $P<0.001)$, but there was no difference in average systolic blood pressure.

Biochemical data in blood of the subjects by nutritional anemia and gender

As shown in Table 3, average serum TC $(P<0.001)$ and serum HDL-C $(P<0.05)$ levels in NA group were significantly lower compared to non-NA group. Average serum TG levels of females were $120.3 \mathrm{mg} / \mathrm{dL}$ in NA group and $141.7 \mathrm{mg} / \mathrm{dL}$ in non-NA group $(P<0.001)$ and average serum LDL-C levels of males were
Table 1. General characteristics of the subjects by nutritional anemia

\begin{tabular}{|c|c|c|c|}
\hline Variables & $\begin{array}{l}\text { NA group } \\
(\mathrm{n}=415)^{1)}\end{array}$ & $\begin{array}{l}\text { Non-NA group } \\
\quad(n=2,843)\end{array}$ & $x^{2}$ or $t-v a l u e$ \\
\hline \multicolumn{4}{|l|}{ Gender } \\
\hline Male & $43.4(2.5)^{2)}$ & $45.2(0.9)$ & \multirow[t]{2}{*}{0.434} \\
\hline Female & $56.6(2.5)$ & $54.8(0.9)$ & \\
\hline Age (yrs) & $73.3 \pm 0.3^{3)}$ & $71.7 \pm 0.1$ & $6.298^{* * * 4)}$ \\
\hline $65-74$ & $56.8(2.6)$ & $70.1(1.0)$ & \multirow[t]{2}{*}{$25.834^{* * *}$} \\
\hline$\geq 75$ & $43.2(2.6)$ & $29.9(1.0)$ & \\
\hline \multicolumn{4}{|l|}{ Residence region } \\
\hline Urban & $73.5(3.1)$ & $73.9(2.3)$ & \multirow[t]{2}{*}{0.024} \\
\hline Rural & $26.5(3.1)$ & $26.1(2.3)$ & \\
\hline \multicolumn{4}{|l|}{ Educational level } \\
\hline$\leq$ Elementary school & $64.4(2.7)$ & $60.2(1.2)$ & \multirow[t]{4}{*}{1.104} \\
\hline Middle school & $12.5(1.9)$ & $13.9(0.8)$ & \\
\hline High school & $16.6(2.1)$ & $17.3(0.8)$ & \\
\hline$\geq$ College & $6.5(1.3)$ & $8.7(0.7)$ & \\
\hline \multicolumn{4}{|l|}{ Income level } \\
\hline Low & $48.6(2.8)$ & $46.4(1.3)$ & \multirow[t]{4}{*}{0.926} \\
\hline Mid-low & $29.0(2.5)$ & $27.4(1.0)$ & \\
\hline Mid-high & $14.1(1.9)$ & $15.7(0.9)$ & \\
\hline High & 8.3 (1.6) & $10.5(0.8)$ & \\
\hline \multicolumn{4}{|c|}{ Number of family members } \\
\hline Single & $26.0(2.4)$ & $19.2(0.8)$ & \multirow[t]{2}{*}{$8.568^{* *}$} \\
\hline$\geq 2$ & $74.0(2.4)$ & $80.8(0.8)$ & \\
\hline \multicolumn{4}{|c|}{$\begin{array}{l}\text { NA: Nutritional Anemia } \\
\text { 1) } N \text { was represented by the unweighted sample size } \\
\text { 2) } \% \text { (SE) } \\
\text { 3) Mean } \pm \mathrm{SE} \\
\text { 4) }{ }^{* \star *} P<0.001 \text {, }{ }^{* *} P<0.01 ; P \text {-values were analyzed by complex sample chi-square } \\
\text { test or complex sample general linear model t-test }\end{array}$} \\
\hline
\end{tabular}

$92.1 \mathrm{mg} / \mathrm{dL}$ in NA group and $107.8 \mathrm{mg} / \mathrm{dL}$ in non-NA group $(P<0.001)$. Average serum creatinine levels in NA group were significantly higher compared to non-NA group in both male $(P<0.05)$ and female $(P<0.001)$.

Major food group intakes of the subjects by nutritional anemia and gender

Major food group intakes of the subjects are shown in Table 4. For males, intakes of potatoes, pulses $(P<0.05)$, and mushrooms $(P<0.01)$ in NA group $(6.8,12.4$, and $0.7 \mathrm{~g}$, respectively) were significantly lower compared to non-NA group $(11.8,19.7$, and

Table 2. Anthropometric and blood pressure data of the subjects by nutritional anemia and gender

\begin{tabular}{|c|c|c|c|c|c|c|}
\hline \multirow[b]{2}{*}{ Variables } & \multicolumn{3}{|c|}{ Male $(n=1,474)^{1)}$} & \multicolumn{3}{|c|}{ Female $(n=1,784)$} \\
\hline & $\begin{array}{l}\text { NA group } \\
(n=182)\end{array}$ & $\begin{array}{l}\text { Non-NA group } \\
\quad(n=1,292)\end{array}$ & t-value & $\begin{array}{l}\text { NA group } \\
(n=233)\end{array}$ & $\begin{array}{l}\text { Non-NA group } \\
\quad(n=1,551)\end{array}$ & t-value \\
\hline Height (cm) & $164.1 \pm 0.5^{2)}$ & $165.3 \pm 0.2$ & $-2.425^{* 3)}$ & $150.1 \pm 0.6$ & $151.7 \pm 0.2$ & $-2.827^{* *}$ \\
\hline Body weight $(\mathrm{kg})$ & $61.1 \pm 0.8$ & $65.0 \pm 0.3$ & $-5.040^{* * *}$ & $53.6 \pm 0.7$ & $56.5 \pm 0.3$ & $-4.088^{* * *}$ \\
\hline Body mass index $\left(\mathrm{kg} / \mathrm{m}^{2}\right)$ & $22.6 \pm 0.3$ & $23.8 \pm 0.1$ & $-4.347^{* * *}$ & $23.8 \pm 0.3$ & $24.5 \pm 0.1$ & $-2.920^{* *}$ \\
\hline Waist circumference $(\mathrm{cm})$ & $82.8 \pm 0.8$ & $86.2 \pm 0.3$ & $-4.254^{* * *}$ & $81.5 \pm 0.8$ & $84.1 \pm 0.3$ & $-3.084^{* *}$ \\
\hline Systolic blood pressure (mmHg) & $125.5 \pm 1.6$ & $126.6 \pm 0.6$ & -0.607 & $129.2 \pm 1.5$ & $129.4 \pm 0.5$ & -0.095 \\
\hline Diastolic blood pressure $(\mathrm{mmHg})$ & $66.2 \pm 0.9$ & $73.1 \pm 0.3$ & $-7.171^{* * *}$ & $68.8 \pm 0.9$ & $72.7 \pm 0.3$ & $-4.258^{* * *}$ \\
\hline
\end{tabular}

NA: Nutritional Anemia

1) $\mathrm{N}$ was represented by the unweighted sample

${ }^{2)}$ Mean $\pm \mathrm{SE}$

3) ${ }^{\star * *} P<0.001,{ }^{* *} P<0.01,{ }^{\star} P<0.05 ; P$-values were analyzed by complex sample general linear model t-test 
Table 3. Biochemical data in blood of the subjects by nutritional anemia and gender

\begin{tabular}{|c|c|c|c|c|c|c|}
\hline \multirow[b]{2}{*}{ Variables } & \multicolumn{3}{|c|}{ Male $(n=1,474)^{1)}$} & \multicolumn{3}{|c|}{ Female $(n=1,784)$} \\
\hline & $\begin{array}{l}\text { NA group } \\
(n=182)\end{array}$ & $\begin{array}{l}\text { Non-NA group } \\
\quad(n=1,292)\end{array}$ & t-value & $\begin{array}{l}\text { NA group } \\
(n=233)\end{array}$ & $\begin{array}{l}\text { Non-NA group } \\
\quad(n=1,551)\end{array}$ & $\mathrm{t}$-value \\
\hline Fasting blood glucose (mg/dL) & $112.4 \pm 3.1^{2)}$ & $107.3 \pm 0.8$ & 1.643 & $104.2 \pm 1.6$ & $106.7 \pm 0.8$ & -1.407 \\
\hline Serum total cholesterol $(\mathrm{mg} / \mathrm{dL})$ & $163.6 \pm 2.9$ & $180.7 \pm 1.1$ & $-5.566^{* * *}$ & $181.4 \pm 2.9$ & $194.8 \pm 1.1$ & $-4.406^{* * *}$ \\
\hline Serum HDL-cholesterol (mg/dL) & $44.5 \pm 0.9$ & $46.5 \pm 0.4$ & $-1.996^{* 3)}$ & $47.1 \pm 0.9$ & $49.4 \pm 0.3$ & $-2.310^{*}$ \\
\hline Serum triglyceride $(\mathrm{mg} / \mathrm{dL})$ & $118.2 \pm 10.6$ & $138.7 \pm 2.6$ & -1.872 & $120.3 \pm 4.3$ & $141.7 \pm 2.3$ & $-4.499 * * *$ \\
\hline Serum LDL-cholesterol (mg/dL) & $92.1 \pm 3.7$ & $107.8 \pm 1.8$ & $-3.912^{* * *}$ & $110.4 \pm 4.1$ & $117.0 \pm 1.4$ & -1.531 \\
\hline Serum creatinine (mg/dL) & $0.77 \pm 0.02$ & $0.73 \pm 0.0$ & $2.201^{*}$ & $0.86 \pm 0.02$ & $0.75 \pm 0.01$ & $5.126^{* * *}$ \\
\hline
\end{tabular}

NA: Nutritional Anemia

1) $\mathrm{N}$ was represented by the unweighted sample

${ }^{2)}$ Mean \pm SE

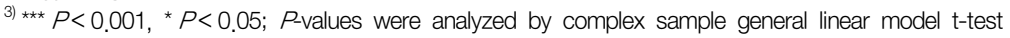

Table 4. Major food group intakes of the subjects by nutritional anemia and gender

\begin{tabular}{|c|c|c|c|c|c|c|}
\hline \multirow[b]{2}{*}{ Variables } & \multicolumn{3}{|c|}{ Male $(n=1,474)^{1)}$} & \multicolumn{3}{|c|}{ Female $(n=1,784)$} \\
\hline & $\begin{array}{l}\text { NA group } \\
(n=182)\end{array}$ & $\begin{array}{l}\text { Non-NA group } \\
\quad(n=1,292)\end{array}$ & t-value & $\begin{array}{l}\text { NA group } \\
(n=233)\end{array}$ & $\begin{array}{l}\text { Non-NA group } \\
\quad(n=1,551)\end{array}$ & t-value \\
\hline Grains (g) & $309.5 \pm 11.0^{2)}$ & $320.7 \pm 4.5$ & -0.962 & $269.6 \pm 9.3$ & $277.9 \pm 4.6$ & -0.797 \\
\hline Potatoes (g) & $6.8 \pm 1.9$ & $11.8 \pm 1.8$ & $-1.972^{* 3)}$ & $5.3 \pm 1.3$ & $14.3 \pm 2.3$ & $-3.512^{* * *}$ \\
\hline Saccharides (g) & $4.8 \pm 0.9$ & $4.3 \pm 0.6$ & 0.552 & $3.0 \pm 0.4$ & $2.6 \pm 0.3$ & -1.312 \\
\hline Pulses (g) & $12.4 \pm 2.7$ & $19.7 \pm 2.5$ & $-2.125^{*}$ & $10.9 \pm 2.6$ & $11.8 \pm 1.3$ & -0.310 \\
\hline Nuts (g) & $3.0 \pm 1.2$ & $4.1 \pm 0.8$ & -0.732 & $1.5 \pm 0.7$ & $3.0 \pm 0.5$ & -1.637 \\
\hline Vegetables (g) & $136.1 \pm 17.6$ & $132.8 \pm 10.7$ & 0.185 & $109.6 \pm 17.8$ & $108.0 \pm 9.0$ & 0.092 \\
\hline Mushrooms (g) & $0.7 \pm 0.3$ & $1.8 \pm 0.4$ & $-2.703^{* *}$ & $0.9 \pm 0.5$ & $1.3 \pm 0.3$ & -0.732 \\
\hline Fruits (g) & $69.8 \pm 14.1$ & $75.5 \pm 8.0$ & -0.367 & $55.0 \pm 10.8$ & $80.8 \pm 8.2$ & $-2.271^{*}$ \\
\hline Seaweed (g) & $14.2 \pm 5.1$ & $15.3 \pm 2.8$ & -0.189 & $6.6 \pm 3.2$ & $14.2 \pm 2.8$ & -1.783 \\
\hline Seasonings (g) & $12.3 \pm 1.8$ & $11.7 \pm 1.1$ & 0.309 & $8.3 \pm 1.5$ & $8.1 \pm 0.7$ & 0.161 \\
\hline Other vegetables $(\mathrm{g})$ & $0.2 \pm 0.1$ & $0.4 \pm 0.2$ & -1.228 & $0.4 \pm 0.4$ & $0.2 \pm 0.1$ & 0.550 \\
\hline Meats (g) & $22.5 \pm 4.8$ & $27.8 \pm 3.7$ & -0.961 & $11.4 \pm 2.7$ & $18.7 \pm 2.2$ & $-2.182^{*}$ \\
\hline Eggs (g) & $5.5 \pm 1.4$ & $6.5 \pm 0.9$ & -0.691 & $2.9 \pm 0.8$ & $4.9 \pm 0.6$ & $-2.269^{*}$ \\
\hline Seafood (g) & $37.4 \pm 10.6$ & $40.0 \pm 4.5$ & -0.238 & $19.2 \pm 5.2$ & $32.7 \pm 4.3$ & $-2.209^{*}$ \\
\hline Milk/dairy products (g) & $16.3 \pm 4.5$ & $14.8 \pm 2.0$ & 0.305 & $28.1 \pm 6.4$ & $21.4 \pm 2.7$ & 1.000 \\
\hline Beverages (g) & $26.2 \pm 6.4$ & $17.4 \pm 2.2$ & 1.382 & $12.4 \pm 3.3$ & $15.3 \pm 1.9$ & -0.793 \\
\hline Alcohol (g) & $40.4 \pm 18.0$ & $31.0 \pm 5.0$ & 0.506 & $5.6 \pm 4.7$ & $3.9 \pm 1.2$ & 0.351 \\
\hline
\end{tabular}

NA: Nutritional Anemia

1) $\mathrm{N}$ was represented by the unweighted sample

2) Mean \pm SE

3) ${ }_{* \star *} P<0.001,{ }^{*} P<0.05$; $P$-values were analyzed by complex sample general linear model t-test

$1.8 \mathrm{~g}$, respectively). For females, intakes of potatoes $(P<0.001)$, fruits, meats, eggs, and seafood $(P<0.05)$ in NA group $(5.3,55.0$, $11.4,2.9$, and $19.2 \mathrm{~g}$, respectively) were significantly lower compared to non-NA group $(14.3,80.8,18.7,4.9$, and $32.7 \mathrm{~g}$, respectively).

Nutrient intakes of the subjects by nutritional anemia and gender

Nutrient intakes of the subjects and proportions of the subjects less than EAR of nutrients by nutritional anemia and gender are shown in Table 5. Energy intake in NA and non-NA groups were $1,785.7$ and $1,991.8 \mathrm{kcal}$ for male subjects $(P<$ $0.001)$ and $1,398.9$ and $1,548.4 \mathrm{kcal}$ for female subjects $(P<0.01)$, respectively. As for differences in intakes of nutrients to support hematopoiesis, in males, significant differences were observed between NA and non-NA groups for intakes of protein (55.7 and $65.8 \mathrm{~g})$, vitamin $B_{2}$ (1.0 and $1.2 \mathrm{mg}$ ), niacin (13.1 and 15.2 $\mathrm{mg} \mathrm{NE})(P<0.001)$, iron (16.2 and $18.3 \mathrm{mg}, P<0.01)$, respectively.
In females, significant differences were observed between NA and non-NA groups for intakes of protein (41.7 and $48.6 \mathrm{~g}$ ), niacin (9.5 and $11.5 \mathrm{mg} \mathrm{NE})$, iron (12.7 and $15.6 \mathrm{mg})(P<0.001)$, vitamin $B_{2}$ (0.8 and $\left.0.9 \mathrm{mg}\right)$, vitamin $C(76.1$ and $102.0 \mathrm{mg})$ $(P<0.01)$, and vitamin A (466.0 and $600.8 \mu \mathrm{gRAE})(P<0.05)$, respectively.

To observe the intakes level of nutrient to support hematopoiesis, the proportion of subjects whose nutrient intakes less than EAR of KDRls was analyzed according to nutritional anemia. In total subjects, the proportion of the subjects whose protein (48.3\% and $36.2 \%)$, vitamin $B_{2}(77.6 \%$ and $64.9 \%)$, niacin $(63.8 \%$ and $49.3 \%)(P<0.001)$, vitamin $A(63.9 \%$ and $56.0 \%, P<0.01)$, and iron $(9.5 \%$ and $5.9 \%, P<0.05)$ intakes less than EAR were significantly higher in NA group compared to non-NA group. Nutrients to support hematopoiesis that were more than $40 \%$ of the proportion of the subjects less than EAR were vitamin $A$, vitamin $B_{2}$, niacin, vitamin $C$, and protein (only in NA group). 


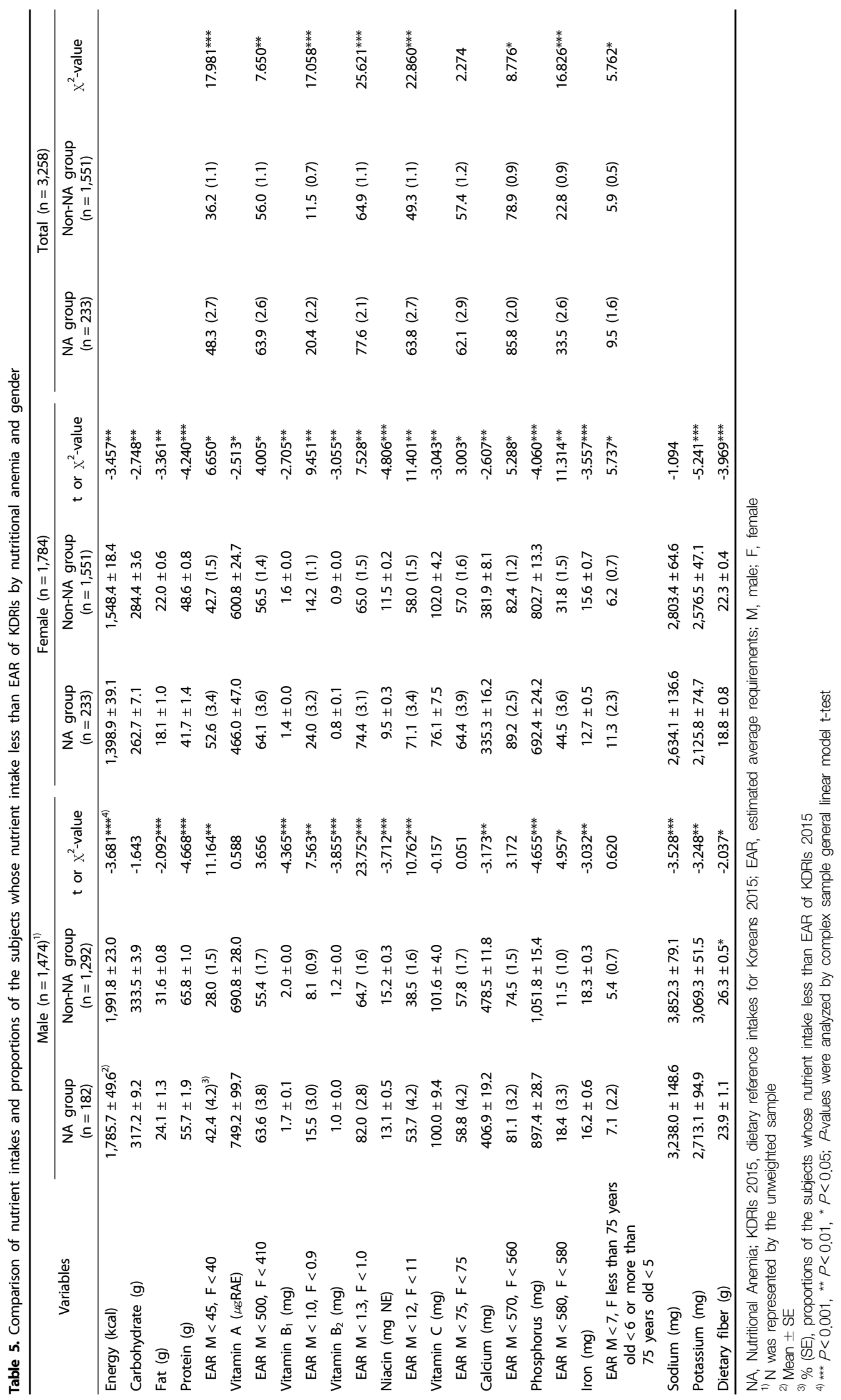


Table 6. Health-related lifestyle habits of the subjects by nutritional anemia and gender

\begin{tabular}{|c|c|c|c|c|c|c|c|c|c|}
\hline \multirow[b]{2}{*}{ Variables } & \multicolumn{3}{|c|}{ Male $(n=1,474)^{1)}$} & \multicolumn{3}{|c|}{ Female $(n=1,784)$} & \multicolumn{3}{|c|}{ Total $(n=3,258)$} \\
\hline & $\begin{array}{l}\text { NA group } \\
(n=182)\end{array}$ & $\begin{array}{l}\text { Non-NA group } \\
\quad(n=1,292)\end{array}$ & $x^{2}$-value & $\begin{array}{l}\text { NA group } \\
(n=233)\end{array}$ & $\begin{array}{l}\text { Non-NA group } \\
\quad(n=1,551)\end{array}$ & $x^{2}$-value & $\begin{array}{l}\text { NA group } \\
(n=415)\end{array}$ & $\begin{array}{l}\text { Non-NA group } \\
\quad(n=2,843)\end{array}$ & $x^{2}$-value \\
\hline \multicolumn{10}{|l|}{ Alcohol drinking ${ }^{2)}$} \\
\hline Yes & $46.2(4.5)^{5)}$ & $59.9(1.7)$ & $8.383^{* * 6)}$ & $14.8(2.7)$ & $18.9(1.2)$ & 1.595 & $27.6(2.5)$ & $37.3(1.0)$ & $10.832^{* * 6)}$ \\
\hline No & $53.8(4.5)$ & $40.1(1.7)$ & & $85.2(2.7)$ & $81.1(1.2)$ & & $72.4(2.5)$ & $62.7(1.0)$ & \\
\hline \multicolumn{10}{|l|}{ Smoking ${ }^{3)}$} \\
\hline Yes & $18.6(3.2)$ & $21.2(1.5)$ & 0.518 & $2.2(1.0)$ & $2.9(0.6)$ & 0.304 & $8.8(1.5)$ & $11.1(0.8)$ & 1.576 \\
\hline No & $81.4(3.2)$ & $78.8(1.5)$ & & $97.8(1.0)$ & $97.1(0.6)$ & & $91.2(1.5)$ & $88.9(0.8)$ & \\
\hline \multicolumn{10}{|c|}{ Walking frequency ${ }^{4)}$} \\
\hline Yes & $7.8(2.1)$ & $6.5(1.0)$ & 0.338 & $10.3(2.5)$ & $6.9(0.9)$ & 2.141 & $9.3(1.8)$ & $6.7(0.7)$ & 2.046 \\
\hline No & $92.2(2.1)$ & $93.5(1.0)$ & & $89.7(2.5)$ & $93.1(0.9)$ & & $90.7(1.8)$ & $93.3(0.7)$ & \\
\hline
\end{tabular}

NA: Nutritional Anemia

${ }^{1)} \mathrm{N}$ was represented by the unweighted sample

2) $<1$ glass/month

3) Current smoking status

4) $\geq 30 \mathrm{~min} /$ day and $\geq 5$ days/week

5) $\%$ (SE)

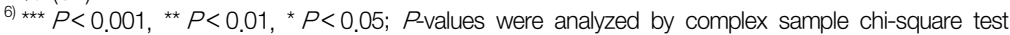

Table 7. Prevalence rate of chronic diseases in the subjects by nutritional anemia and gender

\begin{tabular}{|c|c|c|c|c|c|c|c|c|c|}
\hline \multirow[b]{2}{*}{ Variables } & \multicolumn{3}{|c|}{ Male $(n=1,474)^{1)}$} & \multicolumn{3}{|c|}{ Female $(n=1,784)$} & \multicolumn{3}{|c|}{ Total $(n=3,258)$} \\
\hline & $\begin{array}{l}\text { NA group } \\
(n=182)\end{array}$ & $\begin{array}{l}\text { Non-NA group } \\
\quad(n=1,292)\end{array}$ & $x^{2}$-value & $\begin{array}{l}\text { NA group } \\
(n=233)\end{array}$ & $\begin{array}{l}\text { Non-NA group } \\
\quad(n=1,551)\end{array}$ & $x^{2}$-value & $\begin{array}{l}\text { NA group } \\
(n=415)\end{array}$ & $\begin{array}{l}\text { Non-NA group } \\
\quad(n=2,843)\end{array}$ & $x^{2}$-value \\
\hline \multicolumn{10}{|c|}{ Obesity as determined by BMI (body mass index $\geq 25 \mathrm{~kg} / \mathrm{m}^{2}$ ) } \\
\hline With & $21.3(3.3)^{2)}$ & $32.5(1.5)$ & $8.501^{* * 3)}$ & $33.8(34)$ & $42.2(1.5)$ & $5.050^{*}$ & $28.6(2.6)$ & $37.9(1.0)$ & $11.080^{* *}$ \\
\hline Without & $78.7(3.3)$ & $67.5(1.5)$ & & $66.2(3.4)$ & $57.8(1.5)$ & & $71.4(2.6)$ & $62.1(1.0)$ & \\
\hline \multicolumn{10}{|c|}{ Abdominal obesity (waist circumference $\geq 90 \mathrm{~cm}$ for male and $\geq 85 \mathrm{~cm}$ for female) } \\
\hline With & $24.8(3.7)$ & $33.7(1.6)$ & $4.419^{*}$ & $34.0(3.5)$ & $45.5(1.6)$ & $8.435^{* *}$ & $30.1(2.6)$ & $40.2(1.2)$ & $12.392^{* * *}$ \\
\hline Without & $75.2(3.7)$ & $66.3(1.6)$ & & $66.0(3.5)$ & $54.5(1.6)$ & & $69.9(2.6)$ & $59.8(1.2)$ & \\
\hline \multicolumn{10}{|c|}{ Hypertension (systolic blood pressure or diastolic blood pressure $\geq 140$ or $90 \mathrm{mmHg}$, drug taker) } \\
\hline With & $64.5(4.1)$ & $55.9(1.7)$ & $3.980^{*}$ & $66.4(4.3)$ & $62.2(1.5)$ & 0.915 & $65.6(3.1)$ & $59.4(1.1)$ & $3.987^{*}$ \\
\hline Without & $35.5(4.1)$ & $44.1(1.7)$ & & $33.6(4.3)$ & $37.8(1.5)$ & & $34.4(3.1)$ & $40.6(1.1)$ & \\
\hline \multicolumn{10}{|c|}{ Diabetes (fasting blood glucose $\geq 126 \mathrm{mg} / \mathrm{dL}$, drug taker, insulin injection) } \\
\hline With & $39.9(4.3)$ & $22.0(1.4)$ & $19.492^{* * *}$ & $31.0(3.9)$ & $23.1(1.4)$ & $4.286^{*}$ & $34.7(2.8)$ & $22.6(1.0)$ & $21.093^{* * *}$ \\
\hline Without & $60.1(4.3)$ & $78.0(1.4)$ & & $69.0(3.9)$ & 76.9 (1.4) & & $65.3(2.8)$ & 77.4 (1.0) & \\
\hline \multicolumn{10}{|c|}{ Hypercholesterolemia (serum total cholesterol $\geq 240 \mathrm{mg} / \mathrm{dL}$, drug taker) } \\
\hline With & $18.2(3.7)$ & $17.5(1.2)$ & 0.033 & $29.7(3.5)$ & $35.8(1.4)$ & 2.456 & $25.0(2.7)$ & $27.6(1.0)$ & 0.807 \\
\hline Without & 81.8 (3.7) & 82.5 (1.2) & & $70.3(3.5)$ & $64.2(1.4)$ & & $75.0(2.7)$ & $72.4(1.0)$ & \\
\hline \multicolumn{10}{|c|}{ Hypertriglyceridemia (serum triglyceride $\geq 200$ mg/dL) } \\
\hline With & $8.4(2.5)$ & $15.3(2.5)$ & $4.734^{*}$ & $10.2(2.5)$ & 15.7 (1.1) & 3.223 & $9.4(1.7)$ & $15.5(0.9)$ & $7.997^{* *}$ \\
\hline Without & $91.6(1.3)$ & 84.7 (1.3) & & $89.8(2.5)$ & $84.3(1.1)$ & & $90.6(1.7)$ & $84.5(0.9)$ & \\
\hline \multicolumn{10}{|c|}{ Myocardial infarction or angina pectoris (doctor's diagnosis) } \\
\hline With & $12.8(2.6)$ & $7.0(0.8)$ & $5.593^{*}$ & $9.2(2.5)$ & $6.5(0.8)$ & 1.271 & $10.7(1.8)$ & $6.7(0.5)$ & $5.731^{*}$ \\
\hline Without & $87.2(2.6)$ & $93.0(0.8)$ & & $90.8(2.5)$ & $93.5(0.8)$ & & $89.3(1.8)$ & $93.3(0.5)$ & \\
\hline \multicolumn{10}{|c|}{ Osteoarthritis or rheumatoid arthritis (doctor's diagnosis) } \\
\hline With & $18.0(3.4)$ & $14.1(1.2)$ & 1.277 & $54.7(3.8)$ & $44.8(1.6)$ & $6.051^{*}$ & $39.2(2.9)$ & $31.2(1.1)$ & $7.546^{* *}$ \\
\hline Without & $82.0(3.4)$ & 85.9 (1.2) & & $45.3(3.8)$ & 55.2 (1.6) & & $60.8(2.9)$ & $68.8(1.1)$ & \\
\hline \multicolumn{10}{|c|}{ Kidney failure (doctor's diagnosis) } \\
\hline With & $0(0.0)$ & $0.4(0.2)$ & 1.217 & $0.6(0.4)$ & $0.6(0.2)$ & 0.000 & $0.3(0.2)$ & $0.5(0.1)$ & 0.248 \\
\hline Without & $100.0(0.0)$ & $99.6(0.2)$ & & $99.4(0.4)$ & $99.4(0.2)$ & & $99.7(0.2)$ & $99.5(0.1)$ & \\
\hline
\end{tabular}

NA: Nutritional Anemia

1) $\mathrm{N}$ was represented by the unweighted sample

2) $\%$ (SE)

3) ${ }^{* \star \star} P<0.001,{ }^{* \star} P<0.01,{ }^{*} P<0.05$ by complex sample chi-square test 
Table 8. Odds ratios for nutritional anemia according to chronic diseases

\begin{tabular}{|c|c|c|c|c|}
\hline & \multirow{2}{*}{ Variables } & \multicolumn{3}{|c|}{ Nutritional anemia } \\
\hline & & Model 1 OR $(95 \% \mathrm{Cl})$ & Model 2 OR $(95 \% \mathrm{Cl})$ & Model 3 OR $(95 \% \mathrm{Cl})$ \\
\hline \multicolumn{5}{|c|}{ Obesity as determined by body mass index } \\
\hline \multirow[t]{2}{*}{ Male } & With & $0.56(0.38-0.84)^{* * 1)}$ & $0.61(0.41-0.92)^{*}$ & $0.66(0.43-1.00)$ \\
\hline & Without (ref) & & & \\
\hline \multirow[t]{2}{*}{ Female } & With & $0.70(0.51-0.96)^{*}$ & $0.71(0.52-0.98)^{*}$ & $0.89(0.58-1.36)$ \\
\hline & Without (ref) & & & \\
\hline \multirow[t]{2}{*}{ Total } & With & $0.65(0.51-0.85)^{* *}$ & $0.68(0.52-0.88)^{* *}$ & $0.64(0.49-0.84)^{* *}$ \\
\hline & Without (ref) & & & \\
\hline \multicolumn{5}{|c|}{ Abdominal obesity } \\
\hline \multirow[t]{2}{*}{ Male } & With & $0.65(0.43-0.98)^{*}$ & $0.66(0.43-0.99)^{*}$ & $0.71(0.12-1.10)$ \\
\hline & Without (ref) & & & \\
\hline \multirow[t]{2}{*}{ Female } & With & $0.62(0.44-0.86)^{* *}$ & $0.61(0.43-0.85)^{* *}$ & $0.56(0.40-0.78)^{* *}$ \\
\hline & Without (ref) & & & \\
\hline \multirow[t]{2}{*}{ Total } & With & $0.64(0.50-0.83)^{* *}$ & $0.63(0.49-0.81)^{* * *}$ & $0.60(0.46-0.79)^{* * *}$ \\
\hline & Without (ref) & & & \\
\hline \multicolumn{5}{|c|}{ Hypertension } \\
\hline \multirow[t]{2}{*}{ Male } & With & $1.44(1.00-2.06)^{*}$ & $1.37(0.96-1.97)$ & $1.52(1.03-2.24)^{*}$ \\
\hline & Without (ref) & & & \\
\hline \multirow[t]{2}{*}{ Female } & With & $1.20(0.82-1.77)$ & $1.12(0.76-1.65)$ & $0.86(0.51-1.44)$ \\
\hline & Without (ref) & & & \\
\hline \multirow[t]{2}{*}{ Total } & With & $1.31(1.00-1.71)^{*}$ & $1.22(0.94-1.59)$ & $1.27(0.96-1.67)$ \\
\hline & Without (ref) & & & \\
\hline \multicolumn{5}{|l|}{ Diabetes } \\
\hline \multirow[t]{2}{*}{ Male } & With & $2.36(1.63-3.43)^{* * *}$ & $2.64(1.81-3.86)^{* * *}$ & $2.54(1.71-3.77)^{* * *}$ \\
\hline & Without (ref) & & & \\
\hline \multirow[t]{2}{*}{ Female } & With & $1.50(1.03-2.18)^{*}$ & $1.44(0.99-2.09)$ & $1.36(0.93-1.99)$ \\
\hline & Without (ref) & & & \\
\hline \multirow[t]{2}{*}{ Total } & With & $1.82(1.42-2.34)^{* * *}$ & $1.82(1.42-2.34)^{* * *}$ & $1.74(1.35-2.25)^{* * *}$ \\
\hline & Without (ref) & & & \\
\hline \multicolumn{5}{|c|}{ Hypertriglyceridemia } \\
\hline \multirow[t]{2}{*}{ Male } & With & $0.51(0.26-0.99)^{*}$ & $0.52(0.27-1.03)$ & $0.61(0.31-1.19)$ \\
\hline & Without (ref) & & & \\
\hline \multirow[t]{2}{*}{ Female } & With & $0.61(0.35-1.08)$ & $0.63(0.35-1.12)$ & $0.59(0.33-1.06)$ \\
\hline & Without (ref) & & & \\
\hline Total & With & $0.57(0.37-0.86)^{* *}$ & $0.58(0.38-0.89)^{*}$ & $0.59(0.39-0.91)^{*}$ \\
\hline & Without (ref) & & & \\
\hline Myocardia & or angina pect & & & \\
\hline Male & With & $1.95(1.16-3.31)^{*}$ & $1.81(1.07-3.05)^{*}$ & $1.80(1.04-3.09)^{*}$ \\
\hline & Without (ref) & & & \\
\hline Female & With & $1.46(0.77-2.76)$ & $1.38(0.72-2.64)$ & $1.37(0.71-2.64)$ \\
\hline & Without (ref) & & & \\
\hline Total & With & $1.67(1.12-2.49)^{*}$ & $1.57(1.04-2.37)^{*}$ & $1.59(1.05-2.41)^{*}$ \\
\hline & Without (ref) & & & \\
\hline Osteoarthr & umatoid arthritis & & & \\
\hline Male & With & $1.33(0.82-2.18)$ & $1.21(0.72-2.03)$ & $1.16(0.68-1.98)$ \\
\hline & Without (ref) & & & \\
\hline Female & With & $1.49(1.08-2.05)^{*}$ & $1.42(1.02-1.98)^{*}$ & $1.38(0.99-1.93)$ \\
\hline & Without (ref) & & & \\
\hline Total & With & $1.43(1.11-1.83)^{* *}$ & $1.32(1.01-1.73)$ & $1.21(0.92-1.61)$ \\
\hline & Without (ref) & & & \\
\hline
\end{tabular}

NA, Nutritional Anemia; OR, odds ratio; $\mathrm{Cl}$, confidence interval

Model 1: without adjustment; Model 2: adjusted for age and the number of family members; Model 3: adjusted for age, the number of family members, energy intake, and alcohol drinking

1) ${ }_{\star \star \star} P<0.001,{ }^{\star \star} P<0.01,{ }^{\star} P<0.05$ by Logistic regression for nutritional anemia with chronic diseases 
However, in both groups, the proportion of iron intake was less than $10 \%$ of EAR and was in good intake level.

\section{Health-related lifestyle habits of the subjects by nutritional anemia and gender}

As shown in Table 6, in male subjects, the percentage of alcohol drinking in NA group was significantly lower compared to non-NA group $(P<0.01)$. Percentages of smokers and walking frequency were similar in the two groups; $8.8 \%$ in NA group and $11.1 \%$ in non-NA group were smokers, and $9.3 \%$ in NA group and $6.7 \%$ in non-NA group walked for more than 30 minutes per day for 5 days per week.

Odds ratios for nutritional anemia according to chronic diseases

As shown in Table 7, the prevalence of hypertension $(P<0.05$ in male and total), diabetes $(P<0.001$ in male and total, $P<$ 0.05 in female), myocardial infarction or angina pectoris $(P<$ 0.05 in male and total), and osteoarthritis or rheumatoid arthritis $(P<0.05$ in female, $P<0.01$ in total $)$ in NA group were significantly higher compared to non-NA group. However, the prevalence of obesity as determined by BMI $(P<0.01$ in male and total, $P<0.05$ in female), abdominal obesity $(P<0.05$ in male, $P<0.01$ in female, $P<0.001$ in total), and hypertriglyceridemia $(P<0.05$ in male, $P<0.01$ in total) in NA group were significantly lower compared to non-NA group. There was no significant difference in hypercholesterolemia and kidney failure between NA and non-NA groups.

Except for hypercholesterolemia and chronic kidney failure, the degree of risk exerted by chronic diseases on nutritional anemia is shown in Table 8. In males, for model 1 as risk in crude ORs for chronic diseases, obesity as determined by BMI, abdominal obesity, and hypertriglyceridemia were associated with a decrease in the prevalence of nutritional anemia. After controlling for age and the number of family members (model 2), obesity as determined by BMI and abdominal obesity were significantly decreased the prevalence of nutritional anemia, but age and the number of family members were significantly contributed to hypertriglyceridemia. Whereas in males, for model 1, hypertension, diabetes, and myocardial infarction or angina pectoris were associated with an increase in the prevalence of nutritional anemia. For model 2, diabetes and myocardial infarction or angina pectoris were significantly increased the prevalence of nutritional anemia, but age and the number of family members were significantly contributed to hypertension. After controlling for age, the number of family members, energy intake, and alcohol drinking (model 3), hypertension (1.52 times, $95 \% \mathrm{Cl}=1.03-2.24)$, diabetes $(2.54$ times, $95 \% \mathrm{Cl}=1.71-3.77$ ), myocardial infarction or angina pectoris (1.80 times, $95 \% \mathrm{Cl}=1.04-3.09$ ) were significantly increased the prevalence of nutritional anemia.

In Females, for model 1, obesity as determined by BMI and abdominal obesity were associated with a decrease in the prevalence of nutritional anemia. For model 2, obesity as determined by BMI and abdominal obesity were significantly decreased the prevalence of nutritional anemia. For model 3, abdominal obesity $(0.56$ times, $95 \% \mathrm{Cl}=0.40-0.78)$ was significantly decreased the prevalence of nutritional anemia but age and the number of family members were significantly contributed to obesity as determined by BMI. Whereas in females, for model 1, diabetes and osteoarthritis or rheumatoid arthritis were associated with an increase in the prevalence of nutritional anemia. For model 2, osteoarthritis or rheumatoid arthritis was significantly increased the prevalence of nutritional anemia, but age and the number of family members were significantly contributed to diabetes.

In total subjects, for obesity as determined by BMl $(0.64$ times, $95 \% \mathrm{Cl}=0.49-0.84)$, abdominal obesity $(0.60$ times, $95 \% \mathrm{Cl}=$ $0.46-0.79$ ), and hypertriglyceridemia ( 0.59 times, $95 \% \mathrm{Cl}=0.39$ 0.91) were significantly decreased the prevalence of nutritional anemia. Whereas in total subjects, for model 1, hypertension, diabetes, myocardial infarction or angina pectoris, and osteoarthritis or rheumatoid arthritis were associated with an increase in the prevalence of nutritional anemia. For model 2, diabetes and myocardial infarction or angina pectoris were significantly increased the prevalence of nutritional anemia, but age and the number of family members were significantly contributed to hypertension and osteoarthritis or rheumatoid arthritis. For model 3, diabetes (1.74 times, $95 \% \mathrm{Cl}=1.35-2.25)$ and myocardial infarction or angina pectoris (1.59 times, $95 \% \mathrm{Cl}=1.05-2.41$ ) were significantly increased the prevalence of nutritional anemia.

\section{DISCUSSION}

Nutritional anemia in the elderly is known to be affected by poor quality and/or monotonous diet, gastrointestinal blood loss, and poor nutrient absorption due to demographics and health factors, etc. The purpose of this study was conducted to compare nutritional status according to nutritional anemia and to determine associations between nutritional anemia and chronic diseases in Korean elderly using $6^{\text {th }}$ KNHANES data.

Average age in NA group was two years older than in non-NA group and proportion of the subjects aged $\geq 75$ was also higher. With age, average hemoglobin concentration declined and the prevalence of anemia also increased [4,24]. Furthermore, nutrients utilization is poorer in the elderly due to physiological aging and drugs taking to treat various diseases, and thus, hematologic changes including reductions in hemoglobin concentration may occur $[4,25]$. In this study, the proportion of elderly living alone was significantly higher in NA group than in Non-NA group. The dietary lifestyle of the elderly were closely related to the number of family members, and it was reported that the quality of nutrient intakes by elderly living alone was significantly lower than that of elderly living with family due to low-income levels and high meal costs $[26,27]$. This means that poor nutrient intake due to poor quality and monotonous diet of elderly living alone or getting older may cause nutritional anemia.

In this study, intakes of most foods and nutrients in NA group were significantly lower than in non-NA group, and proportions of the subjects whose nutrient intake less than EAR was also significantly higher in the NA group. Nutritional anemia in the elderly is known to be due to lack of storage of these in blood caused by inadequate intake and incomplete absorption and utilization of hematopoietic nutrients [28]. It was reported that hemoglobin concentration was positively associated with energy and nutrient intakes [29] and there was a difference in blood 
iron and ferritin levels depending on the degree of meat consumption [30]. However, in this study, we found intakes of potatoes, pulses, fruits, meats, eggs, and seafood, which are rich in nutrients for hematopoiesis, were significantly lower in NA group than in non-NA group. In Doyle et al. [31] study, the lower iron storage status in the British aged 65 years and older, intakes of vitamin $C$ and consumption of meat, fish, vegetables, and potatoes were lower. Furthermore, because the consumption of food is reported to be related to masticatory abilities, the elderly with chewing difficulties may be decreased iron storage status. In the elderly with masticatory difficulty, it is possible that food is swallowed without chewing, which would result in poor nutrients absorption due to gastric distress and indigestion. Since nutritional anemia is assessed by the concentration of hemoglobin, ferritin, transferrin, iron, folic acid, and vitamin $B_{12}$ in the blood, foods with high nutrient density should be selected and taken [5]. As a result, it is considered to be related to the lack of nutrient intakes and lack of food (meat, fruit, and egg, etc.) intakes in the elderly, so individualized nutrition education and management should be done on how to eat foods appropriately for deficient nutrient intakes.

Meanwhile, it was reported that nutritional anemia in the elderly accounted for 30 to $40 \%$ of all anemia cases and that iron-deficiency anemia was associated with about two-thirds of nutritional anemia cases [8]. Although iron intake in NA group was significantly lower than in non-NA group, average iron intake of NA group was 2.2 times higher than EAR of KDRIs and proportion of the subjects whose iron intake less than EAR in NA group was $7.1 \%$ of males and $11.3 \%$ of females. In Huang et al. [32] study, Taiwanese elderly were average iron intake more than $10 \mathrm{mg}$ higher than the daily recommended dietary iron intake level, but iron storage level in blood was not significantly associated with iron intake. This is due to a decrease in impaired efficiency of iron absorption in the elderly by causes as food type, aging, the presence of gastrointestinal lesions by chronic disease, etc. [8,33]. Iron bioavailability depends on the chemical nature of the iron and on the kinds of nutrients consumed. In particular, the availability of non-heme iron was influenced by meat, poultry, fish, and vitamin C intakes [34], which are lower in elderly with anemia. In addition, the most common cause of iron deficiency anemia in the elderly is a decrease in hemoglobin due to gastrointestinal blood loss [18]. Therefore, it is necessary to consume balanced hematopoietic nutrients to improve the iron storage in blood, and it is necessary to perform periodic health screening management to check for the occurrence cause of iron deficiency anemia due to chronic diseases.

Alcohol drinking rate of NA group was significantly lower compared to non-NA group $(P<0.01)$. In Teresa et al. [35] study, the degree of alcohol drinking was significantly higher in the group with high hemoglobin concentration and in Yun et al. [36] study, Korean female adults who drink alcohol showed 0.62 times significantly lower anemia prevalence compared to non-drinker. Alcohol can cause a change in iron metabolism, but it can quickly return to normal when drinker stops alcohol drinking [37]. However, chronic drinking can result in gastrointestinal bleeding as gastritis and gastric ulcers, which may lead to iron loss and the result of vitamin $B_{12}$ and folate deficiency [38], so excessive drinking should be avoided.

The subjects of this study showed significant differences according to age, the number of family members, energy intake and alcohol drinking according to nutritional anemia. In previous studies $[39,40]$, these factors were reported to affect anemia. Thus, the ORs of nutritional anemia by chronic diseases was analyzed by adjusting confounding factors as age, the number of family members, energy intake and alcohol drinking.

This study showed that obesity, hypertension, diabetes, hypertriglyceridemia, myocardial infarction or angina pectoris, and osteoarthritis or rheumatoid arthritis were associated with nutritional anemia. As regards obesity as determined by BMI, it was significantly less prevalent in NA group $(21.3 \%$ in males, $33.8 \%$ in females) than in non-NA group (32.5\% in males, $42.2 \%$ in females). Kim et al. [41] reported the prevalence of anemia in Korean elderly was negatively associated with body weight and BMI, and that hemoglobin concentration was positively correlated with BMI. Similarly, in this study, the prevalence of abdominal obesity in NA group $(24.8 \%$ in males, $34.0 \%$ in females) was significantly lower compared to non-NA group (33.7\% in males, $45.5 \%$ in females). In Chinese study, the average waist circumference of elderly women living in rural areas was lower than observed for female subjects in this study, but it was also found average waist circumference was significantly lower in anemic women than in normal controls (75.8 vs. 79.1 $\mathrm{cm}$ ) [42]. In the elderly, BMI exhibits a high positive correlation with nutritional status [43], and BMI loss due to poor nutrient intakes may result in diminished immune function, muscle loss, and possibly nutritional anemia [44]. Therefore, elderly need to consume quality foods rich in protein, vitamins, and minerals and should be careful not to reduce food intake to lose body weight unconditionally.

Analysis showed the prevalence of hypertension, diabetes, myocardial infarction or angina pectoris, and osteoarthritis or rheumatoid arthritis was significantly higher in NA group. The ORs for nutritional anemia according to the presence of diabetes (1.738 times) and of myocardial infarction or angina pectoris (1.590 times) after adjustment appeared significant positive associations. NHANES III (1991-1994) [45] showed the prevalence of diabetes and osteoarthritis was significantly higher in elderly with nutritional anemia than in elderly without nutritional anemia. In the KNHANHS (2013-2014) [19], the OR of nutritional anemia was 1.66 times for the presence of osteoarthritis in Korean elderly $(95 \% \mathrm{Cl}=1.10-2.54)$. For diabetic patients, it was reported to be at high risk for nutritional anemia because hemoglobin concentrations were reduced caused by microvascular disease [46] and diabetic drugs taking were prone to depletion of folic acid and vitamin $B_{12}$ [47]. For osteoarthritis or rheumatoid arthritis patients, clinical symptoms related to anemia may occur because arthritis drugs can inhibit hematopoiesis [48]. For myocardial infarction or angina pectoris patients, an increase in the oxygen demand of the body can lead to nutritional anemia by lower hemoglobin concentrations due to heavy oxygen transport [49]. These findings suggest the elderly need to consume foods rich in iron, vitamins, and protein to prevent nutritional anemia as complications of chronic diseases. And, because more than $30 \%$ of elderly people have more than two chronic diseases [19], if the elderly with chronic diseases 
know the cause of nutritional anemia through doctor's screening, it will be helpful in treatment of nutritional anemia.

This study has limitations. Because data collected for $6^{\text {th }}$ KNHANES on food and nutrient consumption were obtained by the 24-hour recall method, this study could not assess usual daily intake amounts of the subjects. KNHANES is a crosssectional study, which has the disadvantage of not being able to determine the causal relationship, although the association can be suggested. The data of this study dealt with only quantitative intakes, thus there was a limitation in delineating the relationship directly between chewing, digestion, and absorption and quantitative intakes. Thus, further study is required to determine the cause of nutritional anemia. Despite these limitations, this study provides scientific evidence regarding health problems associated with food intakes and relations between chronic diseases and nutritional anemia in the elderly. We believe this study data could be utilized for implementing health promotion related to nutritional anemia and chronic diseases for the elderly in the future.

In summary, our findings suggest nutrient intakes and chronic diseases in Korean elderly are related to the prevalence of nutritional anemia and the elderly with older age or elderly living alone are more likely to the prevalence of nutritional anemia. Nutritional anemia is generally treated by supplementation of nutrients but is diagnosed by biochemical assessment in blood, so the elderly need to choose foods that have high nutrient density and improve the quality of their diet. Therefore, in order to prevent nutritional anemia of Korean elderly, systematic, individualized, nutritional management and education programs that meet their social and economic conditions should be provided and implemented so that hematopoietic nutrients such as iron, protein, and vitamin $B_{2}$ are consumed. In addition, since nutritional anemia due to chronic diseases may occur, additional efforts should be considered to ensure check the elderly for nutritional anemia during the health screening.

\section{CONFLICT OF INTEREST}

The authors declare no potential conflicts of interests.

\section{ORCID}

So Hyun Park: https://orcid.org/0000-0001-5552-8919

So Hee Han: https://orcid.org/0000-0002-7477-1516

Kyung Ja Chang: https://orcid.org/0000-0001-6717-664X

\section{REFERENCES}

1. Seshadri S. Nutritional anemia during pregnancy, early childhood and adolescence: the critical development periods. In: Vir SC, editor. Public Health Nutrition in Developing Countries. New Delhi: WPI Publishing; 2011. p.674-712.

2. United Nations. World Population Ageing. New York (NY): United Nations; 2009.

3. World Health Organization Scientific Group on Nutritional Anaemias; World Health Organization. Nutritional Anaemias: Report of a WHO Scientific Group. Geneva: World Health Organization; 1968.
4. Ershler WB, Sheng S, McKelvey J, Artz AS, Denduluri N, Tecson J, Taub DD, Brant $\sqcup$, Ferrucci L, Longo DL. Serum erythropoietin and aging: a longitudinal analysis. J Am Geriatr Soc 2005;53:1360-5.

5. Stauder R, Thein SL. Anemia in the elderly: clinical implications and new therapeutic concepts. Haematologica 2014;99:1127-30.

6. Guralnik JM, Eisenstaedt RS, Ferrucci L, Klein HG, Woodman RC Prevalence of anemia in persons 65 years and older in the United States: evidence for a high rate of unexplained anemia. Blood 2004;104:2263-8.

7. Patel KV. Epidemiology of anemia in older adults. Semin Hematol 2008;45:210-7.

8. Andrès $E$, Vogel $T$, Zulfiqar A. Anemia in elderly patients: state of art, with a focus on nutritional anemia. In: Means RT Jr, editor. Anemia in the Young and Old. Basel: Springer; 2019. p.179-93.

9. Andrès $E$, Federici $L$, Serraj $K$, Kaltenbach $G$. Update of nutrientdeficiency anemia in elderly patients. Eur J Intern Med 2008;19: 488-93.

10. Park JH, Kwon $\mathrm{HK}, \mathrm{Kim} \mathrm{Bl}, \mathrm{Choi} \mathrm{CH}$, Choi $\mathrm{YH}$. A survey on the oral health condition of institutionalized elderly people resident in free asylum. J Korean Acad Dent Health 2002;26:555-66.

11. Shin SK, Kim HJ, Choi BY, Lee SS. A comparison of food frequency for the elderly regarding different family types: based on Community Health Survey for 2008. Korean J Nutr 2012;45:264-73.

12. Cho SA. The world is getting old ... Is there a country for the elderly? [Internet]. Seoul: Chosun Ilbo Corporation; 2017 [cited 2019 February 15]. Available from: http://news.chosun.com/site/data/ html_dir/2017/04/21/2017042101779.html.

13. Korean Statistical Information Service. Prevalence of anemia [Internet]. Daejeon: Statistics Korea; 2018 [cited 2019 February 15]. Available from: http://kosis.kr/statHtml/statHtml.do?orgld=117\& tblld=DT_11702_N110.

14. e-Country index (KR). Life table, national approval statistics. No. 101035 [Internet]. Daejeon: Statistics Korea; 2018 [cited 2019 February 15]. Available from: http://www.index.go.kr/potal/main/ EachDtIPageDetail.do?idx_cd=2758.

15. Korean Statistical Information Service. Major population indicators [Internet]. Daejeon: Statistics Korea; 2017 [cited 2019 February 15]. Available from: http://kosis.kr/statHtml/statHtml.do?orgld=101\&tblld =DT_1BPA002\&vw_cd=MT_ZTITLE\&list_id=A41_10\&seqNo=\&lang_ mode=ko\&language $=$ kor\&obj_var_id=\&itm_id=\&conn_path $=M T$ _ ZTITLE.

16. Joung $\mathrm{KH}$, Oh $\mathrm{YH}$, Lee $\mathrm{YK}$, Oh MA, Kang $\mathrm{YN}$, Kim KR, Hwang $\mathrm{NH}$, Kim SJ, Lee SH, Lee SG, Hong SY. Survey of the Elderly in 2017. Sejong: Korea Institute for Health and Social Affairs; 2017.

17. e-Medical Information (KR). Women in their forties, should be anemic caution [Internet]. Seoul: e-Medical Information; 2017 [cited 2019 March 6]. Available from: http://www.kmedinfo.co.kr/news/ articleView.html?idxno $=43747$.

18. Jung CW. Anemia in the elderly. Proceedings of the 62th Fall Conference of The Korean Association of Internal Medicine. Seoul: The Korean Association of Internal Medicine; 2011. p.153-6.

19. Kim MJ, Yun JM. The presence or absence of osteoarthritis and related risk factors in Korean elderly using the 6th Korea National Health and Nutrition Examination Survey (2013 2014 KNHANES VI). Korean J Food Nutr 2017;30:995-1006.

20. Ministry of Health and Welfare; The Korean Nutrition Society. Dietary Reference Intakes for Korean. Seoul: The Korean Nutrition Society; 2015. 
21. Rural Development Administration, National Rural Resources Development Institute (KR). Food Composition Table. 9th ed. Jeonju: Rural Development Administration, National Rural Resources Development Institute; 2016.

22. Ministry of Health and Welfare (KR); Korea Centers for Disease Control \& Prevention. Health Behavior and Chronic Disease Statistics 2012. Cheongju: Korea Centers for Disease Control \& Prevention; 2014.

23. Ministry of Health and Welfare (KR); Korea Centers for Disease Control \& Prevention. Korea National Health and Nutrition Examination Survey Raw Data Use Guidelines. Cheongju: Korea Centers for Disease Control \& Prevention; 2017.

24. Tettamanti M, Lucca U, Gandini F, Recchia A, Mosconi $P$, Apolone G, Nobili A, Tallone MV, Detoma P, Giacomin A, Clerico M, Tempia P, Savoia L, Fasolo G, Ponchio L, Della Porta MG, Riva E. Prevalence, incidence and types of mild anemia in the elderly: the "Health and Anemia" population-based study. Haematologica 2010;95:1849-56.

25. Walsh JR. Hematologic disorders in the elderly. West J Med 1981;135:446-54.

26. Lim YJ, Choi YS. Seasonal nutrient intakes of elderly women living alone as compared to those living with family in the Gyeongbuk rural area. Korean J Community Nutr 2007;12:58-67.

27. Bae JS, Kim MH, Kim SB. Effects of nutrition education and personalized lunch service program for elderly at senior welfare center in Jeonju. Korean J Community Nutr 2013;18:65-76.

28. Carmel R. Nutritional anemias and the elderly. Semin Hematol 2008;45:225-34.

29. Lopez-Contreras MJ, Zamora-Portero S, Lopez MA, Marin JF, Zamora $\mathrm{S}$, Perez-Llamas F. Dietary intake and iron status of institutionalized elderly people: relationship with different factors. J Nutr Health Aging 2010;14:816-21.

30. Haider LM, Schwingshackl L, Hoffmann G, Ekmekcioglu C. The effect of vegetarian diets on iron status in adults: a systematic review and meta-analysis. Crit Rev Food Sci Nutr 2018;58:1359-74.

31. Doyle W, Crawley H, Robert H, Bates CJ. Iron deficiency in older people: interactions between food and nutrient intakes with biochemical measures of iron; further analysis of the National Diet and Nutrition Survey of people aged 65 years and over. Eur J Clin Nutr 1999;53:552-9.

32. Huang $Y$, Wong $Y$, Wueng $S$, Cheng $C$, Su K. Nutrient intakes and iron status of elderly men and women. Nutr Res 2001;21:967-81.

33. Rutishauser $I H$, Bates CJ, Paul AA, Black AE, Mandal AR, Patnaik BK. Long-term vitamin status and dietary intake of healthy elderly subjects. 1. Riboflavin. Br J Nutr 1979;42:33-42.

34. Joo E, Kim IS, Seo EA. Nutritional status of iron of elderly in Jeon-Ju area. Korean J Community Nutr 2000;5:493-501.

35. Juárez-Cedillo T, Basurto-Acevedo L, Vega-García S, Manuel-Apolinar
L, Cruz-Tesoro E, Rodríguez-Pérez JM, García-Hernández N, Pérez-Hernández N, Fragoso JM. Prevalence of anemia and its impact on the state of frailty in elderly people living in the community: SADEM study. Ann Hematol 2014;93:2057-62.

36. Yun MJ, Yun ME, Kim MR. Blood nutrition indices and disease diagnosis by anemia in Korean adults. J Korean Soc Food Sci Nutr 2017;46:1443-52.

37. Eichner ER, Hillman RS. Effect of alcohol on serum folate level. J Clin Invest 1973;52:584-91.

38. Lindenbaum J, Lieber CS. Hematologic effects of alcohol in man in the absence of nutritional deficiency. N Engl J Med 1969;281: 333-8.

39. Shi Z, Zhen S, Wittert GA, Yuan B, Zuo H, Taylor AW. Inadequate riboflavin intake and anemia risk in a Chinese population: five-year follow up of the Jiangsu Nutrition Study. PLoS One 2014;9:e88862.

40. Thomson CA, Stanaway JD, Neuhouser ML, Snetselaar LG, Stefanick $\mathrm{ML}$, Arendell $\mathrm{L}$, Chen $\mathrm{Z}$. Nutrient intake and anemia risk in the women's health initiative observational study. J Am Diet Assoc 2011;111:532-41.

41. Kim YK, Lee HO, Chang R, Choue R. A study on the food habits, nutrient intake and the disease distribution in the elderly (aged over 65 years)(I). Korean J Community Nutr 2002;7:516-26.

42. Zhang J, Wang CR, Jin SH, Song PK, Meng LP, Man QQ, Jia SC. Risk factors on anemia among rural elderly women aged 50-75 y in Xinning county, Anhui province, China. Zhonghua Liu Xing Bing Xue Za Zhi 2009;30:227-30.

43. Li HC, Ryu HK. Study on relevance of nutritional status, nutrient intake, and chronic disease risk based on Mini Nutritional Assessment (MNA) of elderly people in Shandong province of China. Korean J Community Living Sci 2017;28:5-16.

44. Kim H, Yoon JL, Lee A, Jung Y, Kim MY, Cho JJ, Ju YS. Prognostic effect of body mass index to mortality in Korean older persons. Geriatr Gerontol Int 2018;18:538-46.

45. Semba RD, Ricks MO, Ferrucci L, Xue QL, Guralnik JM, Fried LP. Low serum selenium is associated with anemia among older adults in the United States. Eur J Clin Nutr 2009;63:93-9.

46. Thomas MC, Tsalamandris C, Maclsaac RJ, Jerums $G$. The epidemiology of hemoglobin levels in patients with type 2 diabetes. Am J Kidney Dis 2006;48:537-45.

47. Lee TH. Depleting nutrients with diabetic drugs taking [Internet]. Seoul: Brunch; 2018 [cited 2019 April 20]. Available from: https:// brunch.co.kr/@4173924/3.

48. Kim HY. Recent trends in rheumatoid arthritis treatment. Korean J Intern Med 1996;51:271-9.

49. Hong TJ. Diagnosis of coronary artery disease. J Korean Med Assoc 2004;47:714-25. 